\title{
Association of disclosure of HIV status with medication adherence
}

\author{
Aurélie Rotzinger ${ }^{a, b}$, Isabella Locatellic, Matthias Reymermier ${ }^{b}$, Sebastian Amico ${ }^{b}$, \\ Olivier Bugnon ${ }^{a, b}$, Matthias Cavassini ${ }^{~}{ }^{*}$, Marie Paule Schneider $^{a, b^{*}}$ \\ a Community pharmacy, School of Pharmaceutical Sciences, University of Geneva, Geneva, Switzerland \\ ${ }^{b}$ Community pharmacy, Department of Ambulatory Care \& Community Medicine, University of Lausanne, \\ Lausanne, Switzerland \\ ${ }^{c}$ Department of Ambulatory care \& Community Medicine, University of Lausanne, Lausanne, Switzerland \\ ${ }^{d}$ Infectious Disease Service, CHUV, University of Lausanne, Lausanne, Switzerland \\ *The last two authors are equal senior contributors
}

\section{Corresponding author:}

Marie P. Schneider, PhD

Community Pharmacy center, Policlinique Médicale Universitaire,

44 Rue du Bugnon, Lausanne 1011, Switzerland

E-mail: marie-paule.schneider@hospvd.ch

Tel: +41.21 .314 .48 .45$

Fax: +41.21 .314 .48 .40$ 


\section{ABSTRACT}

\section{Objective}

Disclosure may affect adherence to antiretroviral treatment. In a medication adherence program, this cross-sectional study describes disclosure, perceived reaction after disclosure, living situations, and the relationship of disclosure with antiretroviral adherence.

\section{Methods}

A combination of a questionnaire to measure disclosure and longitudinal electronic monitoring of medication adherence was used.

\section{Results}

A total of 103 out of 159 eligible patients gave informed consent. The characteristics differed between participants and nonparticipants (race, education, sexual orientation, medication adherence). Thirteen participants did not disclose their HIV status. Seventy-three (81\%) participants judged the reaction after disclosure positive. Among the 62 participants cohabiting, $52 \%$ disclosed to all co-residents. Adherence was high (median 100\%). $\underline{\text { HIV disclosure was }}$ negatively associated with adherence, when disclosing to the mother ( $O R=2.46, p$-value $=0.086$ ) and to siblings ( $\mathrm{OR}=2.89, \mathrm{p}$-value $=0.029$ ). Living alone was associated to a lower adherence than cohabitation (RateRatio $=1.42$, $\mathrm{p}$-value $=0.007$ ).

\section{Conclusion}

HIV disclosure and adherence are sensitive issues, which may explain the reason for refusal. Nonparticipants may be those with the most difficulties disclosing.

\section{Practice implications}


An unbiased collection of sensitive information, as HIV disclosure, is a difficult task. A cohort design, with research data collected systematically by a trusted healthcare provider, may better describe the association between adherence and disclosure.

\section{Keywords}

medication adherence, disclosure, HIV, social support, refusal to participate 


\section{INTRODUCTION}

Disclosure of HIV diagnosis is a complex and emotionally charged decision involving numerous factors, including the length of time since the HIV diagnosis and the patient's social network. Sex partners are often the first to receive disclosure, before friends and family members. Full disclosure to children often occurs slowly as the children age [1-3]. However, not everyone will eventually disclose their HIV status; approximately $10 \%$ of patients will never talk about their medical condition [4].

Prior to disclosure, patients must first balance the positive and negative consequences of this action. These consequences depend on the patient's support system and own emotional goals $[5,6]$. For example, because HIV is still largely stigmatized, disclosure of an HIV-positive status could lead to rejection and stigmatization of a patient, sometimes even by his or her family. In contrast, the patient might desire the honesty and support that may accompany disclosure. Studies in American women have shown that patients who choose to disclose have more social support, less depression, better coping strategies and no regrets regarding the disclosure [7, 8]. The desire to disclose is not solely for personal reasons because the patient is compelled to disclose to protect close relatives and sex partner(s) $[1,2,8]$.

The association between disclosure and medication adherence is not well established in the literature. It is complex and probably mediated by psychological and emotional processes [9-11]. Positive social support and consequently disclosure are both needed to positively impact $\underline{\text { medication adherence [9]. Indeed, poor social support may negatively impact medication }}$ adherence [6]. Likewise if disclosure increases social support, outcomes of HIV infection tend to improve whereas they worsen when support decreases [5]. Similarly, adherence may be more difficult when patients want to hide their status from others, making it difficult at times to take the 
medications as prescribed [12]. Hiding medication or hiding medication intake contribute to treatment interruptions [12].

Taking medication daily is a real challenge for patients on chronic treatments, but it is crucial to reach therapeutic goals. In HIV-positive patients, the daily medication regime entails taking oral combination antiretroviral drugs to suppress the viral load, avoid resistance and prevent the spread of the virus in the community.[13-18] Medication adherence behavior is influenced by a multitude of factors, which are classified by the WHO into 5 different dimensions: patient-related, condition-related, therapy-related, socio-economic and health system-related factors [19, 20]. Disclosure (defined as the process of revealing one's condition) is a patient-related dimension (e.g., influenced by the patient's perception of stigma and mental health), but it is also a condition-related dimension (e.g., disclosing HIV is more complex than disclosing chronic diseases such as hypertension or diabetes). Disclosure is also linked to social factors (e.g., race and society).

To support chronic patients' medication adherence across all 5 WHO dimensions, the pharmacy of the Department of Ambulatory Care \& Community Medicine in Lausanne, Switzerland, implemented a medication adherence program in HIV in collaboration with physicians and nurses in 2004 [21, 22]. In the program, a large number of patients mentioned (among other factors) both the presence or absence of social support and disclosure as a facilitator or barrier to medication adherence. Some patients described taking their antiretroviral treatment only when alone and hiding their treatment in a strategic place (e.g., under a pile of clothes in the rear of a closet, in a locked suitcase under the bed, or in the cellar). Patients also expressed different attitudes regarding disclosure of their HIV condition.

To better comprehend whether our HIV+ cohort population taking part in the adherence program has to hide antiretroviral treatment from others, we systematically describe the extent of 
participant disclosure, including their demographics, living arrangements and perceived reaction towards disclosure, and investigate whether an association exists between medication adherence and HIV disclosure.

\section{METHODS}

\subsection{Study Design and Participants}

We administered a cross-sectional questionnaire among participants of an interdisciplinary medication adherence enhancement program. This program is described elsewhere and explores daily medication adherence through electronic monitoring and brief repeated motivational interviews. It is run by community pharmacists in collaboration with the medical team [23].

All HIV patients who were 18 years and older, fluent in French and had participated for more than 30 days in this interdisciplinary adherence enhancement program between October 2012 and June 2013 were invited to participate.

The protocol was approved by the Ethics committee of Canton de Vaud, Switzerland. Informed consent was obtained from all participants.

\subsection{Procedures:}

After each consecutive routine adherence program visit, a research assistant independent from the clinical staff presented the study to the patient and asked for informed consent. Then, he administered the questionnaire. Reasons for refusing or postponing participation in the study were systematically recorded. Electronic adherence data were extracted from the adherence 
program database. Socio-demographic and clinical data were extracted from the Swiss HIV Cohort Study (SHCS) database.

\section{Measurement}

\section{a.Questionnaire}

The questionnaire was a 10-item survey developed for the purpose of this study because no existing instrument with established psychometric properties was available at the time of the study. The different groups of interest for disclosure were established according to the literature and the SHCS [5, 8, 24-26]. A question was added to explore specifically whether patients had to hide to take their antiretroviral treatment. Eventually, our questionnaire contained 3 dimensions of interest: the extent of disclosure, the attitudes of disclosure recipients and the patient's living situation. For each question, participants had the alternative option of not responding, marking the question as inapplicable, or marking that they did not know. The questionnaire took approximately 10 minutes to complete.

The extent of disclosure was explored through two questions. The first asked "How many people did you disclose to (healthcare professionals excluded)?" (possible answers were 0, 1, 2, 3-5, 610 or $>10)$. The second question reviewed 14 different groups of people to whom participants could have disclosed. For each group, participants indicated if such a group existed within their social environment and then whether they had disclosed to one or more members of the group. The groups included spouses, stable sex partners ( $>6$ months), occasional sex partners, mothers, fathers, siblings, children, other relatives, close friends, religious leaders, colleagues, bosses, acquaintances, and HIV support groups. 
Two questions assessed the attitudes of the disclosure recipients. The first was a general question: "Did people you disclosed to react: always negatively, negatively most of the time, positively most of the time, or always positively?" The second question asked whether the participants' significant others were disclosed to and their current attitude (positive, neutral, or negative).

The living situation included the number and groups of people the participant lived with as well as the percentage of people among those to whom the patient had disclosed his/her HIV status. Possible responses were living alone, with spouse, with sex partner, with children $<18$ years, with relatives, with friends or roommates, or in an institution (e.g., clinic or jail). Participants were permitted to choose more than one answer. Then, the participants were asked about the total number of people living at home and the total number among those people to whom the patient had disclosed.

Because the reaction to disclosure evolves with time, we added a question evaluating the time of first disclosure (<1 month, 1-3 months, 3-6 months, 6-12 months or $>12$ months) to analyze whether the first disclosure occurred recently or in the past and whether the time line differentially impacted adherence. The questionnaire was reviewed by all of the pharmacists of the adherence enhancement program, and changes were made until a consensus was reached. Then, the questionnaire was tested by 3 HIV-positive patients who were not participating in the medication adherence program to verify the comprehension of each item and the time needed for survey administration. Minor changes were made based on the results of this testing.

\section{b.Adherence data}

Because participants were taking part in the adherence enhancement program, adherence data were measured from all of them using electronic monitors (MEMS SmartCap ${ }^{\mathrm{TM}}$, Medication Event Monitoring System, AARDEX, Switzerland) [27]. Each drug of the antiretroviral therapy 
was monitored with a separate monitor. Electronic data were retrieved over the 3-month period preceding the administration of the questionnaire. To increase the reliability of the electronic data, an operational manual described elsewhere (Rotzinger, submitted) was used [28]. Reconciliation using pill counts and interview notes allowed us to add confirmed pocket doses (i.e., pills that the patient removed from the pillbox to swallow later) to the electronic adherence data.

\section{Analysis}

Sociodemographics and adherence data were compared between participants and non participants. To explore the relationship between medication adherence, disclosure and attitudes towards disclosure, we dichotomized the following variables with non-normal distributions: medication adherence ( 0 vs. $\geq 1$ day without intake over the analyzed period), number of disclosure recipients ( 0 vs. $\geq 1$ ), attitudes of disclosure recipients (positive vs. negative), and percentages of disclosure recipients living in the same house $(100 \%$ vs. $<100 \%$; participants living alone were considered $100 \%$ ). Adherence data were analyzed for the last 30 days to link actual adherence with the cross-sectional measurement of disclosure. These analyses were performed using the Stata Statistical Software ${ }^{\mathrm{TM}}$ (release 13, StataCorp LP, TX, USA). The Chi$\underline{\text { square and Wilcoxon sign-rank tests were used when appropriate and calculated with a }}$ significance threshold of $\mathrm{p}=0.05$.

Then, a logistic model was adopted in order to study the association between disclosure (dichotomized into «no disclosure » and « disclosed to at least one person ») and sociodemographic characteristics: age, sex, and ethnicity. A Poisson model with offset and a hurdle component capturing the excess of zeros was used in order to describe the ratio between number of missed doses and total expected doses in a month and its association with sociodemographic and disclosure variables. The Hurdle Poisson model showed a strongly better 
performance in terms of AIC (Akaike Information Criterion) compared to the simpler Poisson model, because of the large percentage of zeros contained in variable "Number of missed doses" The Hurdle Poisson model is composed of two parts: the hurdle component describes the probability of missing at least one dose (binomial model with logistic link), while a truncated Poisson component describes the (positive) number of missed doses over the total expected doses for the 1-month period (offset). A first model was estimated including socio-demographic characteristics: age, sex, and race. Six different models were run, which included as an explanatory variable: 1) disclosure, 2) disclosure to the mother, 3) disclosure to siblings, 4) disclosure to cohabitants, 5) recipient attitude, and 6) significant other's attitude. All such models were adjusted for the socio-demographic characteristics. These analyses were performed using $\underline{\mathrm{R} \text { software package, version 3.2.2 (R: A language and environment for statistical computing. } \mathrm{R}}$ Foundation for Statistical Computing, Vienna, Austria, URL: http://www.R-project.org).

\section{RESULTS}

A total of 159 patients met the inclusion criteria between October 2012 and June 2013, and 103 (65\%) gave informed consent, but 1 was excluded as he did not use the electronic monitors consistently (see Figure 1). Among the 31 patients who explicitly refused the study, 19 (62\%) did so because they did not want to talk about disclosure.

Socio-demographic and clinical data from the 102 participants and 56 nonparticipants (i.e., patients who refused or postponed the study) are summarized in Table 1. We noted several significant differences in socio-demographic and adherence data between the groups. Compared to the participants, the nonparticipants were mainly black ( $58 \%$ vs. $32 \%, p=0.017$ ), had earned a basic educational degree (62\% vs. 30\%, $p=0.001)$, were heterosexual $(89 \%$ vs. 
$67 \%, p=0.008)$ and were more likely to be nonadherent ( $44 \%$ vs. $23 \%, p=0.011)$. There was no difference in clinical data except for the time since diagnosis (median time was 10 years (IC95: $7,16)$ for nonparticipants vs. 7 (IC95: 2,11) for participants). Interestingly, the nonparticipants were also more likely to have refused participation in the Swiss HIV Cohort Study (32\% vs. $10 \%$, $\mathrm{p}=<0.001)$ compared to the participants. Among the participants who had disclosed, all but 5 patients disclosed more than 6 months ago.

A total of 300 pocket doses were added during the reconciliation of the adherence data, which represented $1 \%$ of all doses. Fifty-five (55\%) participants had no missed dose, $22(22 \%)$ had 1 day without intake, and $23(23 \%)$ had more than 1 day without intake (see Table 1). Adherence results over the 3-month period were similar to the one-month period.

Thirteen (13\%) participants reported not having disclosed their HIV status. Among those who had disclosed, 27 (31\%) disclosed to 1 person, $19(21 \%)$ to $2-5$ persons, $15(17 \%)$ to $6-10$ persons and $27(31 \%)$ to more than 10 persons. White participants tended to disclose more often than non-whites $(95 \%(55 / 58)$ vs. $76 \%(25 / 33) \mathrm{p}=0.007)$. Disclosure to different groups ranged from $96 \%(53 / 55)$ of participants disclosing to their spouses to $20 \%(11 / 54)$ disclosing to their bosses (Figure 2).

Among the 88 participants who disclosed, recipients of disclosure were perceived to have positive attitudes by 73 participants (83\%) and negative attitudes by 11 participants (13\%). Among the 80 participants who disclosed to their significant others, their significant others were perceived to have positive attitudes by 74 participants (92\%), neutral attitudes by 4 participants (5\%) and negative attitudes by 2 participants (3\%).

Sixty-three participants (63\%) lived with other people; of them, $34(55 \%)$ disclosed to all of the people they lived with. The participants lived mainly with their spouses (34 participants, 55\%) 
and children (23 participants, 37\%). Among the participants who disclosed to less than $100 \%$ of their co-inhabitants, $18(64 \%)$ had children vs. $5(7 \%)$ of those who disclosed to $100 \%$ of their co-inhabitants $(p<0.001)$.

In a logistic model including socio-demographic characteristics, the probability of disclosing to at least one person was significantly lower when the patient's race belonged to the non-white category $(\mathrm{OR}=0.07, \mathrm{p}$-value $=0.004)$. This probability was lower for men compared to women $(\mathrm{OR}=0.25 \mathrm{p}$-value=0.098) (see Table 2). In a Hurdle Poisson model including socio-demographic characteristics, the probability of missing at least one dose was not significantly related to predictors (hurdle component), while the positive number of missed doses (over the total expected monthly doses) was significantly related to race and age (the latter significant at $10 \%$ ). Namely, the 'missed/expected doses' ratio was $40 \%$ lower in non-white participants $(p<0.001)$ and $11 \%$ higher for each 10 years of age $(p=0.058)$.

$\underline{\text { In a Hurdle Poisson model adjusted for socio-demographic characteristics, disclosure to at least }}$ one person was not associated with the probability of missing at least one dose (Hurdle component: $\mathrm{OR}=0.61, \mathrm{p}$-value $=0.447$ ) (see Table 2). However, when at least one dose is missed, disclosure was significantly associated with a larger number of missed doses, with respect to the total expected monthly doses (truncated Poisson component: Rate Ratio=1.96, $\mathrm{p}$ value $=0.003$ ). Disclosure to the mother and to the siblings acted in the same direction: the estimated 'missed/expected doses' ratio was $85 \%$ higher in those who disclosed to the mother vs. those who did not ( $p$-value $<0.001$ ); the same ratio was $76 \%$ higher in those who disclosed to siblings vs. those who did not ( $\mathrm{p}$-value $=0.001)$. Disclosure to the mother and siblings was also positively associated with the probability of missing at least one dose (Hurdle component:

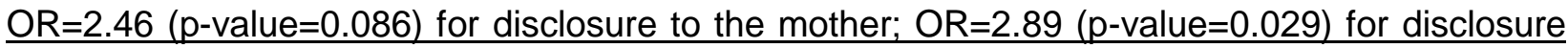
to the siblings). Living alone was associated to a significantly higher 'missed/expected doses' 
ratio than cohabitation, whether the disease was disclosed to all co-residents or not (Rate $\underline{\text { Ratio }=1.42, p \text {-value }=0.007 \text {, with respect to the reference category "disclosed to all co- }}$ residents"). A positive attitude of the recipients was associated with $29 \%$ lower 'missed/expected doses' ratio with respect to the case of a negative attitude ( $p$-value=0.010). However, not disclosing was associated to a lower ratio with respect to disclosing, whether the attitude of recipients was positive or negative (Rate Ratio $=0.43$, $p$-value $<0.001$ ). Disclosure to the significant other and attitude of the significant other seemed to act in the opposite direction, but this result was only significant at $10 \%$.

\section{DISCUSSION AND CONCLUSION}

\subsection{Discussion}

Disclosure often comes into discussions in our medication adherence program because it affects the way patients self-manage their treatment at home and therefore possibly impacts adherence. Patients taking part in this adherence program were recommended by their physicians because they either had or were at risk for adherence issues [21]. The aim of this study was to describe the extent of disclosure of this population and to investigate its association with medication adherence measured electronically and longitudinally in a cohort study. Such knowledge is of importance to healthcare providers addressing medication adherence with their patients within their practices.

Although the acceptance rate of our study (65\%) is satisfying and slightly higher than the $59 \%$ inclusion rate of another HIV disclosure study using a questionnaire, the reasons for refusal are 
mainly because the patients did not want to discuss disclosure (62\% of refusals)[29]. This reason is closely related to our study outcome and raises the question of selection bias and representation. However, because the patients were in a cohort database, we were able to conduct analyses comparing participants and non-participants. The participants in our study were significantly different from the nonparticipants; black race, lower education, heterosexual orientation and most importantly lower adherence were associated with refusal. Among the participants, the results showed that non-whites disclosed less than whites, and men disclosed less than women. They also showed that the probability of missing a larger number of doses is significantly higher in non-white participants vs. whites, and such a probability decreased with age. Interestingly, race, lower education and heterosexual orientation (except lower adherence, which has been less investigated to date), have also been associated with less disclosure in other studies [4, 30, 31]. In our study, we were able to show that adherence was lower among non-participants but was not associated with an increased viral load. Generally, these differential findings between patients who consented to participate in the research versus patients who did not consent to participate are an important topic to elucidate why people agree or do not agree to participate in HIV-related research and the way such self-selection influences the generalizability of the results. Indeed, despite this issue of representation, our rate of nondisclosure $(13 \%)$ is similar to rates described in the literature for other developed countries: in the US, Simoni et al. found a $12 \%$ proportion of nondisclosure, and the same proportion was found by Elford et al. in the UK and by Elopre et al. with patients entering HIV care [2, 4, 31]. This finding leads us to question the representativeness of these other studies because our results demonstrate the difficulty in studying disclosure rates in the HIV+ population.

Half of our participants disclosed to some but not all of their co-residents. However, the high rate of disclosure to stable sex partners (96\%) is encouraging and matches the results of other studies performed in developed countries. In contrast, studies in developing countries show 
other disclosure patterns (namely, disclosure to family members is preferred over disclosure to sex partners) $[1,32,33]$. Disclosure rates are different with occasional sex partners; only $35 \%$ of participants disclosed their status to such partners. This percentage again matches the percentages found in the literature (between $25 \%$ and $50 \%$ according to a review) [30]. Questions concerning sex partners and disclosure are sensitive, and social desirability certainly influences the answers [34]. Participants might hide the fact that they have an occasional partner or pretend that they have disclosed to that partner. To minimize this risk of bias, interviewers were independent from the clinical staff, asked questions in a non-judgmental way, reinforced the fact that the data collection was anonymous, and always gave the possibility of skipping a question. However, trust could positively influence the way patients answer such questions, with some patients being more inclined to reveal true behavior to their usual caregiver.

We hypothesized that non-disclosure would negatively impact adherence because adherence seems to be more difficult for patients who must hide their medication and medication-taking activity [12]. However, our results showed preliminary counterintuitive evidence. Indeed, the probability of missing a larger number of doses increased with total disclosure, and in particular, disclosure to the mother and disclosure to the siblings. Moreover, not disclosing decreased the probability of missing a larger number of doses in comparison to disclosure, whatever the attitude of the recipients. On the contrary, living alone, which could be an indicator of social isolation, increased this probability vs. cohabitation whatever the disclosure status. Disclosure is known to have different possible consequences depending on the types of players involved (e.g., family members and friends) [35]. Our counter-intuitive findings may reveal that people who did not disclose to close family members put more pressure on themselves in term of their own strict control of the infection (and hence strict adherence) compared to people who disclosed, and who are less socially pressured to hide their status and allow themselves to skip a dose once in a while without clinical consequences. Due to their counter-intuitive 
characteristics, these findings warrant further attention and investigation. In another study, disclosure to the mother but not to the father had a positive impact on CD4 counts and viral loads, but only in cases with high family support in non-Hispanic white vs. Latino men [5].

This study has some limitations. First, the rate of medication adherence in our population was very high, which could contribute to a ceiling effect. From a statistical viewpoint, it likely decreased the variability on both the adherence and the disclosure measures, which might have led to a lower observed relationship between these two primary variables of interest. It also explains why most participants were undetectable. Moreover, our convenient sample size was limited by the number of participants in the adherence program and only allowed us to detect a difference of $30 \%$ in the proportion of participants with $\geq 1$ and $<1$ day without intake. Medication adherence is a complex behavior that is influenced by many factors, and studies with larger sample sizes will allow strengthening the evidence. Second, the cross-sectional design did not allow us to consider the temporality of disclosure. Third, we did not investigate the support system provided by each group (specifically the support provided by the mother); additionally, we did not measure perceived stigma, mental health status, emotional and/or practical social support resulting from disclosure, or the impact of self-disclosure vs. uncontrolled disclosure on adherence $[6,8-11,25,35]$. These choices were made to specifically investigate the association between the actual disclosure rate (especially disclosure to co-residents) and HIV medication adherence. A fourth limitation was the absence of validation of our questionnaire as we have not performed any reliability testing yet. This will be performed later on to increase the use and dissemination of an English version of the questionnaire. However, the measured rates of disclosure to stable sex partners and occasional partners are similar to the rates published in the literature, which is in favor of the validity of our results. This study showed that both the disclosure and adherence dimensions are complex. The multiple possible interplays between both dimensions (e.g., disclosing to the mother and siblings) warrant further qualitative 
exploration to answer the questions raised by the article, especially why participants decide to disclose to important people in their lives. Because disclosure and medication adherence are sensitive issues, a cohort design may also better describe the association between medication adherence and disclosure.

\subsection{Conclusion}

Although our study showed that the majority of people disclosed and had a positive experience with disclosure, HIV disclosure remains a sensitive issue in Europe and may explain the reason for refusal and possible selection bias. Indeed, the participants in our study who refused to participate may be those with the most difficulty disclosing and adhering to their antiretroviral treatment.

\subsection{Practice implications}

Disclosure and its impact on adherence are sensitive issues, which healthcare providers should be aware of. Addressing this topic while respecting a patient's integrity is a real challenge. A medication adherence program with a trusted health provider could establish an optimal framework to allow patients to divulge more sensitive information and to allow professionals to better capture a patient's status regarding disclosure and adherence. 
Acknowledgements: The authors would like to thank all of the pharmacy staff for their participation in this study, all of the participants in the present study and the participants in the Swiss HIV Cohort Study because part of the data collected in this study was financed within the framework of the Swiss HIV Cohort Study supported by the Swiss National Science Foundation (grant \# 134277). The authors have no source of funding to declare. 


\section{REFERENCES}

[1] Sowell RL, Seals BF, Phillips KD, Julious CH. Disclosure of HIV infection: how do women decide to tell? Health Educ Res. 2003;18:32-44.

[2] Kalichman SC, DiMarco M, Austin J, Luke W, DiFonzo K. Stress, social support, and HIV-status disclosure to family and friends among HIV-positive men and women. J Behav Med. 2003;26:315-32.

[3] Serovich JM, Craft SM, Reed SJ. Women's HIV Disclosure to Family and Friends. AIDS patient care and STDs. 2012;26:241-9.

[4] Elford J, Ibrahim F, Bukutu C, Anderson J. Disclosure of HIV status: the role of ethnicity among people living with HIV in London. Journal of acquired immune deficiency syndromes. 2008;47:514-21.

[5] Fekete EM, Antoni MH, Lopez CR, Duran RE, Penedo FJ, Bandiera FC, et al. Men's serostatus disclosure to parents: associations among social support, ethnicity, and disease status in men living with HIV. Brain, behavior, and immunity. 2009;23:693-9.

[6] Ushie BA, Jegede AS. The paradox of family support: concerns of tuberculosis-infected HIV patients about involving family and friends in their treatment. AIDS patient care and STDs. 2012;26:674-80.

[7] Serovich JM, McDowell TL, Grafsky EL. Women's report of regret of HIV disclosure to family, friends and sex partners. AIDS and behavior. 2008;12:227-31.

[8] Simoni JM, Demas P, Mason HRC, Drossman JA, Davis ML. HIV Disclosure Among Women of African Descent: Associations with Coping, Social Support, and Psychological Adaptation. AIDS Behav. 2000;4:147-58.

[9] Waddell EN, Messeri PA. Social support, disclosure, and use of antiretroviral therapy. AIDS and behavior. 2006;10:263-72.

[10] Woodward EN, Pantalone DW. The role of social support and negative affect in medication adherence for HIV-infected men who have sex with men. J Assoc Nurses AIDS Care. 2012;23:388-96.

[11] Huynh AK, Kinsler JJ, Cunningham WE, Sayles JN. The role of mental health in mediating the relationship between social support and optimal ART adherence. AIDS Care. 2013.

[12] Katz IT, Ryu AE, Onuegbu AG, Psaros C, Weiser SD, Bangsberg DR, et al. Impact of HIV-related stigma on treatment adherence: systematic review and meta-synthesis. Journal of the International AIDS Society. 2013;16:18640.

[13] Bangsberg DR, Moss AR, Deeks SG. Paradoxes of adherence and drug resistance to HIV antiretroviral therapy. The Journal of antimicrobial chemotherapy. 2004;53:696-9.

[14] Lima VD, Geller J, Bangsberg DR, Patterson TL, Daniel M, Kerr T, et al. The effect of adherence on the association between depressive symptoms and mortality among HIV-infected individuals first initiating HAART. AIDS. 2007;21:1175-83.

[15] Bangsberg DR, Perry S, Charlebois ED, Clark RA, Roberston M, Zolopa AR, et al. Non-adherence to highly active antiretroviral therapy predicts progression to AIDS. AIDS. 2001;15:1181-3.

[16] Buscher A, Hartman C, Kallen MA, Giordano TP. Validity of self-report measures in assessing antiretroviral adherence of newly diagnosed, HAART-naive, HIV patients. HIV clinical trials. 2011;12:24454.

[17] Cambiano V, Lampe FC, Rodger AJ, Smith CJ, Geretti AM, Lodwick RK, et al. Use of a prescriptionbased measure of antiretroviral therapy adherence to predict viral rebound in HIV-infected individuals with viral suppression. HIV medicine. 2010;11:216-24. 
[18] Kahana SY, Rohan J, Allison S, Frazier TW, Drotar D. A meta-analysis of adherence to antiretroviral therapy and virologic responses in HIV-infected children, adolescents, and young adults. AIDS and behavior. 2013;17:41-60.

[19] Sabate E. Adherence to long-term therapies: evidence for action. In: World Health O, editor. Geneva: World Health Organisation; 2003. p. 196.

[20] Kardas P, Lewek P, Matyjaszczyk M. Determinants of patient adherence: a review of systematic reviews. Frontiers in pharmacology. 2013;4:91.

[21] Krummenacher I, Cavassini M, Bugnon O, Schneider MP. An interdisciplinary HIV-adherence program combining motivational interviewing and electronic antiretroviral drug monitoring. AIDS Care. 2011;23:550-61.

[22] Lelubre M, Kamal S, Genre N, Celio J, Gorgerat S, Hugentobler Hampai D, et al. Interdisciplinary Medication Adherence Program: The Example of a University Community Pharmacy in Switzerland. BioMed research international. 2015;2015:ID 103546.

[23] Krummenacher I, Cavassini M, Bugnon O, Schneider MP. Characteristics of HIV patients referred to a medication adherence program in Switzerland. International journal of clinical pharmacy. 2012;34:42631.

[24] Simoni JM, Mason HR, Marks G, Ruiz MS, Reed D, Richardson JL. Women's self-disclosure of HIV infection: rates, reasons, and reactions. Journal of consulting and clinical psychology. 1995;63:474-8.

[25] Rice E, Comulada S, Green S, Arnold EM, Rotheram-Borus MJ. Differential disclosure across social network ties among women living with HIV. AIDS and behavior. 2009;13:1253-61.

[26] Fernandez L, Bonnet A, Guinard A, Pedinielli J-L, Préau M. Outil de receuil d'informations sur les cofacteurs dans l'étude des effets psychosociaux des catastrophes 2005.

[27] Krummenacher I, Cavassini M. Antiretroviral Adherence-Enhancing Management Program in HIV patients (AMP-HIV): A Feasibility Study in the Swiss HIV Cohort Study. (Sent for publication). 2010.

[28] Rotzinger A, Cavassini M, Bugnon O, Schneider MP. Underuse of electronic drug monitors in HIV routine care: its impact on medication adherence data. Submitted. 2014.

[29] Peretti-Watel P, Spire B, Pierret J, Lert F, Obadia Y. Management of HIV-related stigma and adherence to HAART: evidence from a large representative sample of outpatients attending French hospitals (ANRS-EN12-VESPA 2003). AIDS Care. 2006;18:254-61.

[30] Sullivan KM. Male self-disclosure of HIV-positive serostatus to sex partners: a review of the literature. J Assoc Nurses AIDS Care. 2005;16:33-47.

[31] Elopre L, Westfall AO, Mugavero MJ, Zinski A, Burkholder G, Hook EW, et al. Predictors of HIV Disclosure in Infected Persons Presenting to Establish Care. AIDS and behavior. 2015.

[32] Spire B, Bouhnik AD, Obadia Y, Lert F, group Vs. Concealment of HIV and unsafe sex with steady partner is extremely infrequent. AIDS. 2005;19:1431-3.

[33] Longinetti E, Santacatterina M, El-Khatib Z. Gender Perspective of Risk Factors Associated with Disclosure of HIV Status, a Cross-Sectional Study in Soweto, South Africa. PLoS One. 2014;9:e95440.

[34] McCallum EB, Peterson ZD. Investigating the impact of inquiry mode on self-reported sexual behavior: theoretical considerations and review of the literature. Journal of sex research. 2012;49:21226.

[35] Dima AL, Stutterheim SE, Lyimo R, de Bruin M. Advancing methodology in the study of HIV status disclosure: The importance of considering disclosure target and intent. Soc Sci Med. 2014;108:166-74. 


\section{LEGENDS}

Figure 1: Inclusion Flow Chart

Figure 2: Disclosure by Group

Disclosure rates were calculated as follows: number of disclosure recipients / (number of disclosure recipients + number of persons not disclosed to). 
Table 1: Socio-demographic and clinical characteristics of participants who accepted or refused/postponed the questionnaire

\begin{tabular}{|c|c|c|c|c|c|}
\hline \multirow[b]{2}{*}{ Patients not included in SHCS } & \multicolumn{2}{|c|}{$\begin{array}{l}\text { Patients who } \\
\text { accepted } \\
n=102\end{array}$} & \multicolumn{2}{|c|}{$\begin{array}{l}\text { Patients who } \\
\text { refused or } \\
\text { postponed } \\
n=56\end{array}$} & \multirow[t]{2}{*}{$\begin{array}{l}\text { p-value } \\
<0.001^{*}\end{array}$} \\
\hline & 10 & $(10 \%)$ & 18 & $(32 \%)$ & \\
\hline $\begin{array}{l}\text { Socio-demographic data } \\
\text { available }\end{array}$ & $\mathrm{n}=92$ & & $n=38$ & & \\
\hline Gender & & & & & \\
\hline - Men & 51 & $(55 \%)$ & 18 & $(47 \%)$ & 0.402 \\
\hline Age (years) [median, IQR] & 42 & {$[35,51]$} & 42 & {$[35,47]$} & 0.601 \\
\hline Race Whito & & & & & \\
\hline $\begin{array}{l}\text { - White } \\
\text { - Black }\end{array}$ & 58 & $(63 \%)$ & 14 & $(37 \%)$ & \\
\hline $\begin{array}{l}-\quad \text { Black } \\
\text { Educational degree }\end{array}$ & 29 & $(32 \%)$ & 22 & $(58 \%)$ & $0.017^{*}$ \\
\hline - Basic & 27 & $(30 \%)$ & 23 & $(62 \%)$ & \\
\hline - Medium/High & 64 & $(70 \%)$ & 14 & $(38 \%)$ & $0.001^{*}$ \\
\hline Sexual preference & & & & & \\
\hline $\begin{array}{c}-\quad \text { Heterosexual } \\
\text { Source of HIV infection }\end{array}$ & 60 & $(67 \%)$ & 34 & $(89 \%)$ & $0.008^{\star}$ \\
\hline - Sexual contact & 80 & $(87 \%)$ & 32 & $(84 \%)$ & \\
\hline - Drugs & 6 & $(7 \%)$ & 4 & $(11 \%)$ & 0.721 \\
\hline Clinical data & $\mathrm{n}=92$ & & $\mathrm{n}=\mathbf{3 8}$ & & \\
\hline Viral load (copies per ml x 1000) & 0 & {$[0,40]$} & 0 & {$[0,0]$} & 0.299 \\
\hline $\begin{array}{l}\text { Number with undetectable viral } \\
\text { load }\end{array}$ & 63 & $(68 \%)$ & 29 & $(76 \%)$ & 0.372 \\
\hline $\mathrm{CD}_{4}\left(\mathrm{cellls} / \mathrm{mm}^{3}\right)$ & 585 & {$[474,772]$} & 580 & {$[378,784]$} & 0.695 \\
\hline Time since diagnosis (years) & $\begin{array}{l}7 \\
n=81\end{array}$ & {$[2,11]$} & $\begin{array}{l}10 \\
n=30\end{array}$ & {$[7,16]$} & $0.027^{\star}$ \\
\hline $\begin{array}{l}\text { Number of drugs } \\
\text { (antiretrovirals/patient) } \\
\text { Antiretroviral }\end{array}$ & $\begin{array}{l}4 \\
n=87\end{array}$ & {$[3,4]$} & $\begin{array}{l}4 \\
n=34\end{array}$ & {$[3,4]$} & 0.982 \\
\hline$-P I$ & $44 / 87$ & $(51 \%)$ & $31 / 34$ & $(62 \%)$ & 0.267 \\
\hline - NNRTI & $48 / 87$ & $(55 \%)$ & $16 / 34$ & $(47 \%)$ & 0.460 \\
\hline - Other & $22 / 87$ & $(25 \%)$ & $6 / 34$ & $(18 \%)$ & 0.370 \\
\hline $\begin{array}{l}\text { Time in adherence program } \\
\text { (days) }\end{array}$ & $\begin{array}{l}747 \\
n=103\end{array}$ & {$[317,1429]$} & $\begin{array}{l}782 \\
n=32\end{array}$ & {$[522,1310]$} & 0.361 \\
\hline 1-month adherence data ${ }^{a}$ & $\mathrm{n}=99$ & & $\mathrm{n}=50$ & & \\
\hline Number of days without intake & & & & & $0.008^{*}$ \\
\hline $\begin{array}{ll}\text { - } & 0 \text { days } \\
\text { - } & 1 \text { day } \\
\text { - } & >1 \text { day }\end{array}$ & $\begin{array}{l}55 \\
22 \\
22\end{array}$ & $\begin{array}{l}(55 \%) \\
(22 \%) \\
(22 \%)\end{array}$ & $\begin{array}{r}24 \\
4 \\
22\end{array}$ & $\begin{array}{l}(48 \%) \\
(8 \%) \\
(44 \%)\end{array}$ & \\
\hline
\end{tabular}

SHCS = Swiss HIV Cohort Study, PI=Protease inhibitor, NNRTI=Non-nucleoside reverse transcriptase inhibitor, ART=antiretroviral therapy; ${ }^{a} 1$-month adherence prior to study acceptance/refusal, or prior to the end of inclusion period for participants who postponed. Among the patients who postponed participation, 4 patients had quit the adherence program by the time of their enrolment, and 2 patients made many pocket doses, thus invalidating their electronic data. 
Table 2: Logistic and Hurdle Poisson models for association between disclosure, adherence, and socio-demographic characteristics.

Outcome: disclosure

Logistic model:

to $>=1$ person

Odds ratio p-value

Socio-demographic $(n=91)$

Race

White ethnicity

Ref Ref

Other etnicities

0.07

0.004

Gender

Women

Ref

0.25

0.87

Men

$0.87 \quad 0.647$

Age (10 years)

Outcome: number of missed doses in 1 month

Hurdle component: zero missed versus at least one missed dose

Poisson component: (positive) number of missed doses Odds ratio $p$-value Rate ratio $p$-value

\section{Race}

Socio-demographic $(n=90)$

White ethnicity

Ref

Other etnicities

0.95

Ref

Ref

Ref

Gender

Women

Ref

0.915

0.60

$<0.001$

Men

0.56

Ref

Ref

Ref

Age (10 years)

1.20

0.239

0.93

0.551

Total disclosure $(n=89)$ *

No disclosure

Ref

0.371

1.11

0.058

Disclosed to $>=1$ person

0.61

Ref

Ref

Ref

Disclosure to the mother $(n=62)$ *

No disclosure to the mother

Ref

0.447

1.96

0.003

Disclosed to the mother

2.46

Ref

Ref

Ref

Disclosure to siblings ( $\mathrm{n}=75$ ) *

No disclosure to siblings

Ref

0.086

1.85

$<0.001$

Disclosed to siblings

2.89

Ref

Ref

Ref

Disclosed to coresidents $(\mathbf{n = 8 7})$ *

Disclosed to all coresidents

Ref

0.029

1.76

0.001

Did not disclose to all coresidents

0.89

Ref

Ref

Ref

Living alone

1.42

0.825

1.04

0.835

Recipient attitude $(\mathrm{n}=86)$ *

Negative attitude

Ref

0.500

1.42

0.007

Positive attitude

0.54

Ref

Ref

Ref

No disclosure

1.04

0.200

0.71

0.010

Significant other's attitude $(n=82)$ *

Negative attitude of significant other

Ref

0.956

0.43

0.000

Ref

Ref

Ref 


\begin{tabular}{lllll} 
Positive attitude of significant other & 1.21 & 0.842 & 2.96 & 0.096 \\
No disclosure to significant other & 0.75 & 0.794 & 3.16 & 0.096 \\
\hline
\end{tabular}

* Adjusted for socio-demographic characteristics: gender, age, and race 